\title{
Como el hipermercado. Nueva interfaz de Pubmed.
}

Molina Arias M.

Hospital Infantil Universitario La Paz. Madrid. España.

\section{Resumen}

La National Library of Medicine anunció en octubre de 2019 la renovación de su buscador, Pubmed, que convivirá durante unos meses con la plataforma clásica que, finalmente, desaparecerá. Repasamos sus novedades y las bases de su funcionamiento.

\section{Introducción}

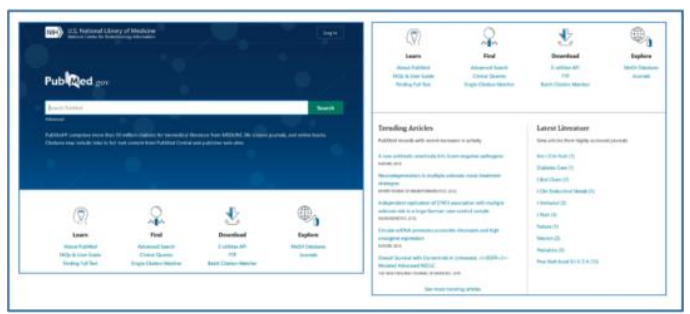

La National Library of Medicine anunció en octubre de 2019 la renovación de su buscador, Pubmed, que convivirá durante unos meses con la plataforma clásica que, finalmente, desaparecerá. Repasamos sus novedades y las bases de su funcionamiento.

ay una cosa que me ocurre de forma recurrente y que me sienta a cuerno quemado. Resulta que a mí me gusta hacer la compra una vez a la semana, así que suelo ir todos los viernes al hipermercado. Yo soy un animal de costumbres que come siempre las mismas cosas y casi los mismos días, así que voy raudo y veloz por los pasillos del hiper echando cosas en el carro y termino de comprar en un santiamén. El problema es que en los hipermercados tienen la mala costumbre de cambiar periódicamente los productos de sitio, con lo que uno se vuelve loco hasta que se lo aprende otra vez. Por si esto fuera poco, los primeros días han cambiado las cosas, pero no los carteles, con lo que tengo que dar mil vueltas hasta encontrar las latas de calamares en su tinta que, como todos sabemos, forman parte de la base de la alimentación actual.

Os preguntaréis por qué os cuento todo este rollo. Pues resulta que la National Library of Medicine (NML) ha hecho una cosa parecida: ahora que por fin había conseguido aprender cómo funcionaba el buscador, van y lo cambian completamente.

Claro que hay que decir en honor de la NML que no se han limitado a cambiar las cajas de ventana, sino que ha implementado un cambio radical con una interfaz que definen como más limpia y sencilla, además de mejor adaptada a los dispositivos móviles, cada vez más utilizados para hacer las búsquedas bibliográficas. Pero ahí no queda la cosa: hay un montón de mejoras en los algoritmos para buscar los más de 30 millones de citas que incluye Pubmed $\mathrm{y}$, además, la plataforma se aloja en la nube, con lo que promete ser más estable y eficiente. 
La NLM anunció el nuevo Pubmed en octubre de 2019 y será la opción por defecto a primeros del año 2020 así que, aunque le versión legacy estará disponible unos meses más, no nos queda más remedio que aprender a manejar la nueva versión. Echemos un vistazo.

Aunque todas las funcionalidades que conocemos de la versión antigua están también presentes en la nueva, el aspecto es radicalmente diferente desde la página de inicio, que os muestro en la primera figura.

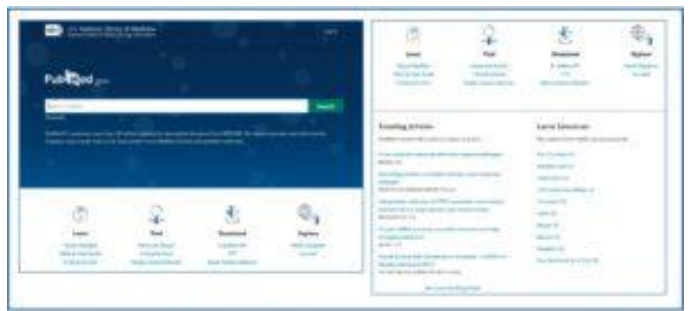

Figura 1. Página de inicio.

El elemento más importante es la nueva caja de búsqueda, donde tenemos que introducir el texto para pulsar seguidamente sobre el botón "Search". Si la NLM no nos engaña, este será el único recurso que tendremos que utilizar la inmensa mayoría de las veces, aunque seguimos teniendo a nuestra disposición un enlace para entrar en el modo de búsqueda avanzada.

Más abajo tenemos cuatro apartados, entre ellos el que contiene ayuda para aprender a usar la nueva versión, y que incluyen herramientas que ya conocíamos, como "Clinical Queries", "Single Citation Matcher" o "MeSH Database". En el momento de escribir esta entrada, estos enlaces te dirigen a las versiones antiguas de las herramientas, pero esto cambiará cuando la nueva interfaz sea a la que se acceda por defecto.

Por último, más abajo se ha añadido un componente nuevo llamado "Trending Articles". Aquí se muestran artículos de interés, que no tienen por qué ser los más recientes, sino aquellos que han despertado interés últimamente y se han podido viralizar de una $u$ otra forma. Junto a esto tenemos la sección de "Latest Literature", donde se muestran artículos recientes de revistas de alto impacto.

Veamos ahora un poco cómo se hacen las búsquedas con el nuevo Pubmed. Una de las claves de esta actualización es la caja de búsqueda simple, que se ha vuelto mucho más lista al incorporar una serie de nuevos sensores que, según la NLM, tratan de detectar exactamente qué es lo que queremos buscar a partir del texto que hemos introducido.

Por ejemplo, sin introducimos información sobre el autor, la abreviatura de la revista y el año, el sensor de citación detectará que hemos introducido información básica de citación y tratará de encontrar el artículo que estamos buscando. Por ejemplo, si yo escribo "campoy jpgn 2019", obtengo los resultados que se ven en la figura 2 , donde se muestran los dos trabajos que Pubmed encuentra de esta doctora publicados en este Journal en 2019. Sería algo parecido a lo que antes obteníamos utilizando el "Single Citation Matcher".

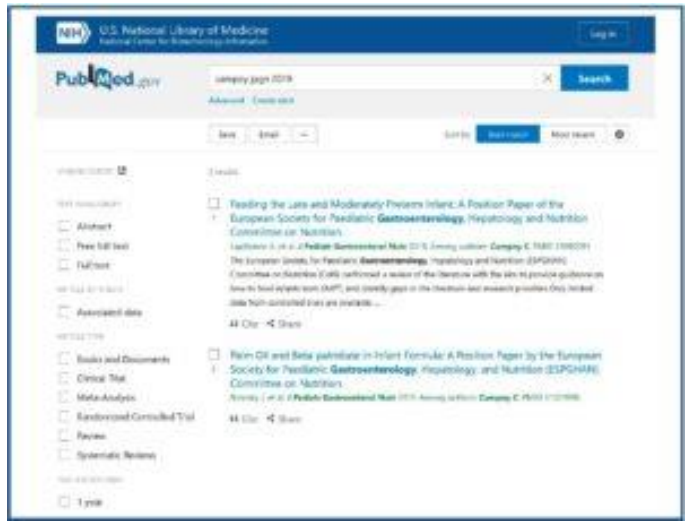

Figura 2. Búsqueda por datos de citación.

También podemos hacer la búsqueda de forma más tradicional. Por ejemplo, si queremos buscar por autor, lo más recomendable es escribir el apellido 
seguido de la inicial del nombre, todo en minúsculas, sin etiquetas ni signos de puntuación. Por ejemplo, si queremos buscar trabajos de Yvan Vandenplas, escribiremos "vandenplas y", con lo que obtendremos los trabajos que os muestro en la figura 3. Por supuesto, también podemos buscar por tema. $\mathrm{Si}$ escribo "parkinson" en la caja de búsqueda, Pubmed me hará una serie de sugerencias sobre los términos de búsqueda parecidos. Si pulso "Search", obtengo los resultados de la figura 4 que, como veis, incluye todos los resultados con los términos relacionados.

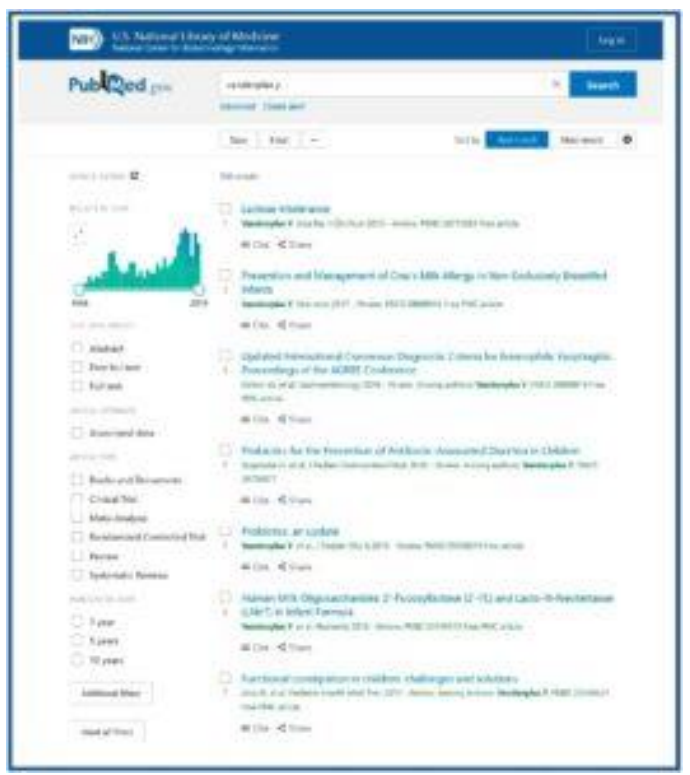

Figura 3. Búsqueda por autor.

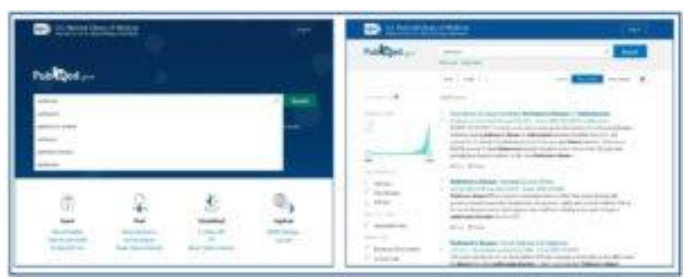

Figura 4. Introducción de términos de búsqueda y pantalla de resultados.

Pasemos ahora a la página de resultados, que también está llena de sorpresas. Podéis ver un detalle en la figura 5. Debajo de la caja de búsqueda tenemos dos enlaces: "Advanced", para acceder a la búsqueda avanzada, y "Create alert", para que Pubmed nos avise cada vez que se incorpore un nuevo artículo relacionado con esta búsqueda (ya sabéis que para esto tenemos que abrir cuenta en NCBI y entrar pulsando el botón "Login" de la parte superior; esta cuenta es gratuita y guarda toda nuestra actividad en Pubmed para usos posteriores).

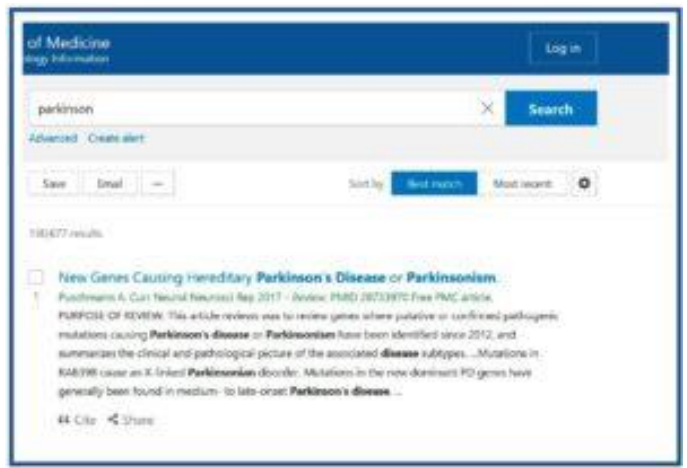

Figura 5. Nuevas funcionalidades de la página de resultados.

Debajo de estos enlaces tenemos tres botones que nos permiten guardar la búsqueda ("Save"), enviarla por correo electrónico ("Email") y, dentro de los tres puntos, enviarla al portapapeles o a nuestra bibliografía o colecciones, si tenemos cuenta en NCBI.

A la derecha tenemos los botones para ordenar los resultados. El "Best Match" es una de las nuevas prioridades de la NLM, que intenta mostrarnos en las primeras posiciones los trabajos más relevantes. De todas formas, podemos ordenarlos por orden cronológico ("Most recent"), al igual que cambiar la forma de presentarlos pulsando sobre la rueda dentada de la derecha (en formato "Summary" o "Abstract").

Pasamos a la izquierda de la página de resultados. Lo primero que vemos es un gráfico con los resultados indexados por año. Este gráfico puede ampliarse, lo que nos permite ver la evolución del número de trabajos sobre el tema indexados a lo largo del tiempo. Además, podemos modificar el intervalo temporal y restringir la búsqueda a lo publicado en un periodo 
determinado. En la figura 6 os muestro como limitar la búsqueda a los resultados de los 10 últimos años.

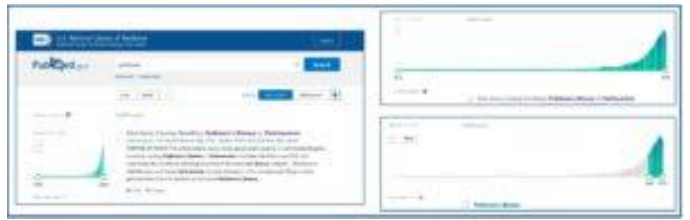

Figura 6. Filtrado por año de publicación.

Debajo de cada resultado tenemos dos enlaces nuevos: "Cite" y "Share". El primero nos permite escribir la cita del trabajo en varios formatos diferentes. El segundo, compartirlo en redes sociales.

Por último, a la izquierda de la pantalla de resultados tenemos el listado de filtros que podemos aplicar. Estos pueden añadirse o quitarse de forma similar a cómo se hacía con la versión antigua de Pubmed y su funcionamiento es muy intuitivo, así que no le vamos a dedicar más tiempo.

Si pulsamos sobre uno de los artículos de la lista de resultados accederemos a la pantalla con el texto del mismo (figura 7). Esta pantalla es similar a la de la versión clásica de Pubmed, aunque se incluyen botones nuevos como "Cite" y los de acceso a redes sociales, además de información adicional sobre artículos relacionados y artículos en los que se cita el que hemos seleccionado. También como novedad, tenemos unas flechas de navegación en los extremos izquierdo y derecho de la pantalla para pasar el texto de los artículos anterior y posterior, respectivamente.

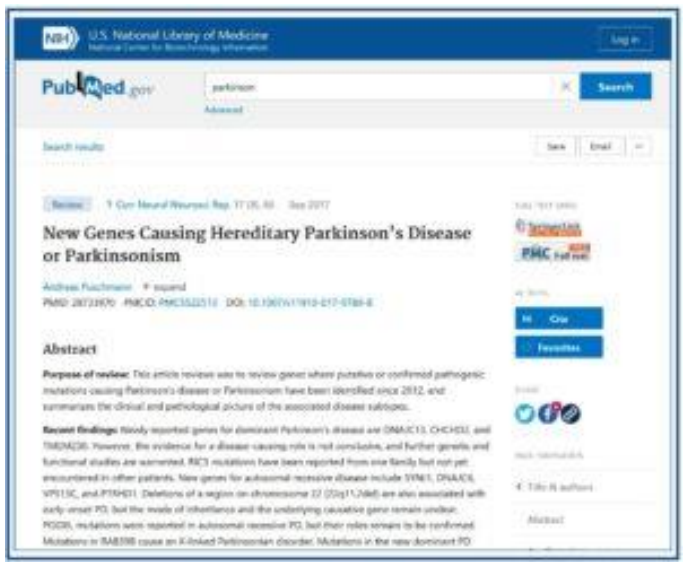

Figura 7. Página resumen del artículo encontrado.

Para ir acabando esta entrada, vamos a echar un vistazo a la nueva búsqueda avanzada, a la que podemos acceder pulsando sobre el enlace "Advanced", que nos llevará a la pantalla que veis en la figura 8 .

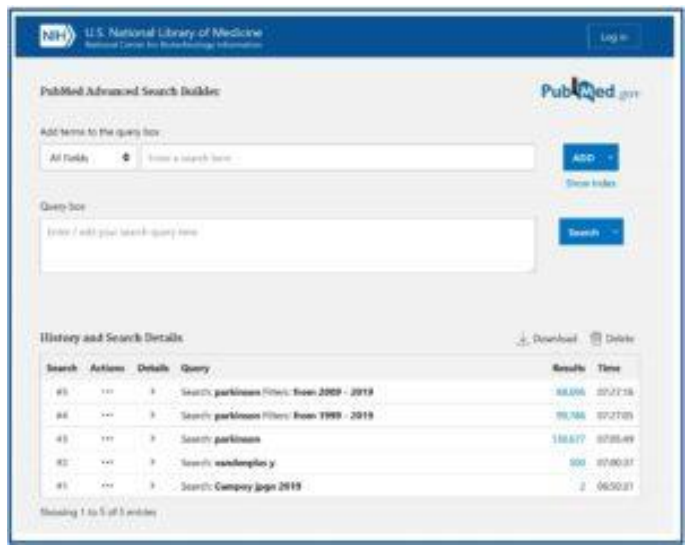

Figura 8. Pantalla de búsqueda avanzada.

El funcionamiento es muy similar al de la versión clásica. Podemos ir añadiendo términos con los operadores booleanos, combinar búsquedas, etc. Os animo a que juguéis con la búsqueda avanzada, las posibilidades son infinitas. La parte más novedosa de esta herramienta es la sección con la historia y los detalles de búsqueda ("History and Search Details"), en la parte inferior. Esto permite conservar búsquedas previas y volver a ellas, teniendo en cuenta siempre que todo esto se borra al salir de Pubmed si no tenemos cuenta en NCBI. 
Llamo vuestra atención sobre la pestaña "Search Details", que podéis abrir tal como os muestro en la figura 9. La búsqueda se hace más transparente, ya que nos muestra cómo la ha interpretado Pubmed en base a un sistema automático de elección de términos ("Automatic Term Mapping"). Aunque nosotros no sepamos muy bien cómo acotar la búsqueda a términos específicos de la enfermedad de Parkinson, Pubmed sí que sabe sobre qué estamos buscando e incluye todos los términos en la búsqueda, además de la cadena inicial que nosotros introducimos, claro está.

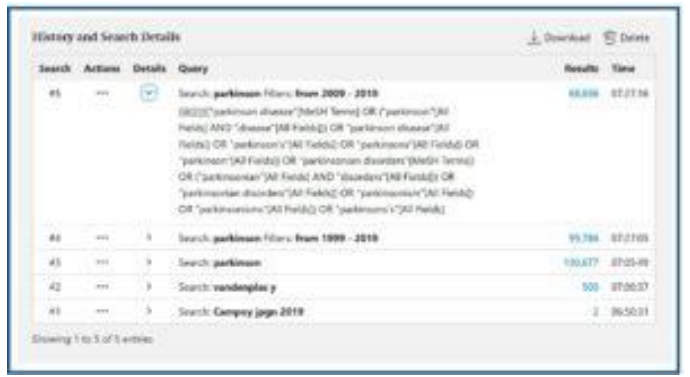

Figura 9. Historia y detalles de búsquedas.

Y aquí acabamos por hoy. Habéis podido ver que estos de la NLM se han superado, poniendo a nuestra disposición una nueva herramienta más sencilla de utilizar, pero, a la vez, mucho más potente e inteligente. Google debe estar temblando, pero nos os preocupéis, seguro que inventa algo para superarse.

Ya podéis ir dejando la versión vieja, no esperéis a que desaparezca para poder empezar a disfrutar de la nueva. Tendremos que volver a hablar de estos temas cuando se establezcan las nuevas versiones del resto de las herramientas, como las Clinical Queries, pero esa es otra historia...

\section{Bibliografía}

- NIH. US National Library of Medicine. A new Pubmed: highlights for information professionals. Disponible en:

www.nlm.nih.gov/oet/ed/pubmed/events/2019 09.html. Consultado el 25/11/2019.

Correspondencia al autor

Manuel Molina Arias mma1961@gmail.com

Servicio de Gastroenterología.

Hospital Infantil Universitario La Paz.

Madrid. España.

Aceptado para el blog en diciembre de 2019 